\title{
MK-801 inhibits the development of morphine tolerance at spinal sites
}

\author{
Howard B. Gutstein ${ }^{a, b, *}$, Keith A. Trujillo ${ }^{b}$ \\ ${ }^{a}$ Department of Anesthesiology, ${ }^{b}$ Mental Health Research Institute, University of Michigan, Ann Arbor, MI 48109-0800, USA
}

(Accepted 27 July 1993)

Key words: Opiate tolerance; MK-801; Spinal cord; Antinociception; Tail flick test; NMDA

\begin{abstract}
The $N$-methyl-D-aspartate (NMDA) receptor antagonist MK- 801 has been shown to attenuate tolerance development in rats. In this study, we show that MK-801 inhibits tolerance to the antinociceptive effects of morphine, as assessed by the tail-flick test, in spinalized rats. These results suggest that NMDA receptor antagonists inhibit opiate tolerance at spinal sites, and also provide strong evidence that the effects of MK-801 are not due to its ability to interfere with associative learning, but instead to inhibition of non-associative mechanisms of opiate tolerance.
\end{abstract}

Opiates are an important class of drugs in the clinical treatment of pain. However, tolerance to the analgesic effects of these drugs develops with chronic use, resulting in decreased effectiveness over time. An important research goal has been to understand the mechanisms underlying the development of opiate tolerance. Such an understanding might lead to treatments that could prevent the development of tolerance and thereby extend the clinical effectiveness of opiate analgesics.

Recent studies in our laboratory ${ }^{13-15}$ and others ${ }^{2,11,12}$ have shown that the non-competitive $N$-methyl-Daspartate (NMDA) receptor antagonist MK-801 (dizocilpine) inhibits the development of opiate tolerance and dependence without affecting pain responsiveness, and without altering the acute analgesic effects of morphine. These results suggest that the development of opiate tolerance and dependence, like other forms of neural and behavioral plasticity, such as learning, long-term potentiation and kindling, involves NMDA receptor activation ${ }^{4,5}$.

Two possible explanations have been suggested for the ability of NMDA receptor antagonists to inhibit opiate tolerance ${ }^{2,14,16,17}$. First, it is possible that NMDA receptor antagonists interfere with the fundamental physiologic changes that occur during the development of tolerance and thereby inhibit non-associative or pharmacologic tolerance. However, learning mechanisms have been found to have a role in the development of tolerance under certain experimental conditions, and NMDA receptor antagonists have been reported to interfere with learning. It is thus possible that NMDA receptor antagonists could inhibit opiate tolerance by interfering with the learning of associations between drug effects and environmental cues, and therefore inhibit associative or behavioral tolerance.

In the present study we had two goals: (1) we were interested in determining whether NMDA receptor antagonists inhibit opiate tolerance by interfering with associative or non-associative mechanisms of tolerance; and (2) we wished to determine whether this effect involved neural changes at the spinal level or in higher brain centers. To accomplish these, we examined the ability of MK-801 to inhibit the development of tolerance to the antinociceptive effects of morphine, as assessed by the tail-flick test, in spinalized rats. The tail-flick response is a spinal reflex that remains intact following spinal transection ${ }^{3}$. Morphine inhibits the tail-flick response in spinalized animals (i.e. it produces antinociception), and tolerance develops to these antinociceptive effects during chronic administration of 
morphine. ${ }^{3}$ Since learning processes, such as the establishment of associations between drug effects and environmental stimuli, presumably require integration at higher brain centers, the spinalized animal appears to be an ideal system for examining a relatively pure non-associative tolerance. If MK-801 interferes with tolerance to the antinociceptive effects of morphine in the spinal animal, then this would suggest that NMDA receptor antagonists have the ability to inhibit non-associative tolerance. In addition, such a finding would indicate that the spinal cord is an important site of action for the inhibition of tolerance by MK- 801 .

Preliminary reports of this work were presented at the 1992 meetings of the American Society of Anesthesiologists ${ }^{8}$ and the Society for Neuroscience ${ }^{9}$.

Institutional approval was obtained prior to the study. Twenty adult male Sprague-Dawley rats (201$225 \mathrm{~g}$, Charles River, Portage, MI, USA) had ad libitum access to food and water, and were maintained on a $12 \mathrm{~h}$ light cycle. Animals were allowed to habituate to the animal colony for 1 week, then underwent a $T_{8}$ spinal transection as previously described ${ }^{7}$. Briefly, under deep chloral hydrate anesthesia, a laminectomy was performed at $T_{8}$ and two segments (approx. $4 \mathrm{~mm}$ ) of spinal cord were completely removed by excavation. Animals were then housed individually in plastic cages for the duration of the study. After allowing one week for recovery from surgery, baseline tail-flick latencies were determined. Animals were then implanted with either one morphine (75 mg, NIDA) or placebo pellet and one osmotic minipump (Alzet, Model 2002, Palo Alto, CA) infusing either MK-801 $(0.1 \mathrm{mg} / \mathrm{kg} /$ day $)$ or saline. On day 3 , one additional pellet of the same type was implanted in each animal. Tail flick latencies ${ }^{6}$ were measured $4 \mathrm{~h}$ after each pellet implantation and daily thereafter. Completeness of the spinal cord lesions was verified using an operating microscope after sacrifice. Data were converted to percent maximum possible effect ${ }^{10}$ and analyzed using a two-factor repeated measures ANOVA. Subsequently, the Fisher PLSD was used as a post-hoc test to analyze differences between groups at specific time points. $P<0.05$ was required for significance.

MK-801, in the presence of placebo pellets, had no effect on tail flick latency when compared with salineinfused control animals (Fig. 1). Rats treated with morphine pellets and saline infusions showed nearmaximal morphine antinociception at $4 \mathrm{~h}$ after the first pellet implant, and developed complete tolerance within $48 \mathrm{~h}$. No antinociceptive effects of morphine were seen in these animals after the second pelleting. In contrast, rats treated chronically with morphine and MK-801 displayed significant levels of morphine antinociception throughout the 6 days of treatment, including an increase in antinociception after the second pelleting.

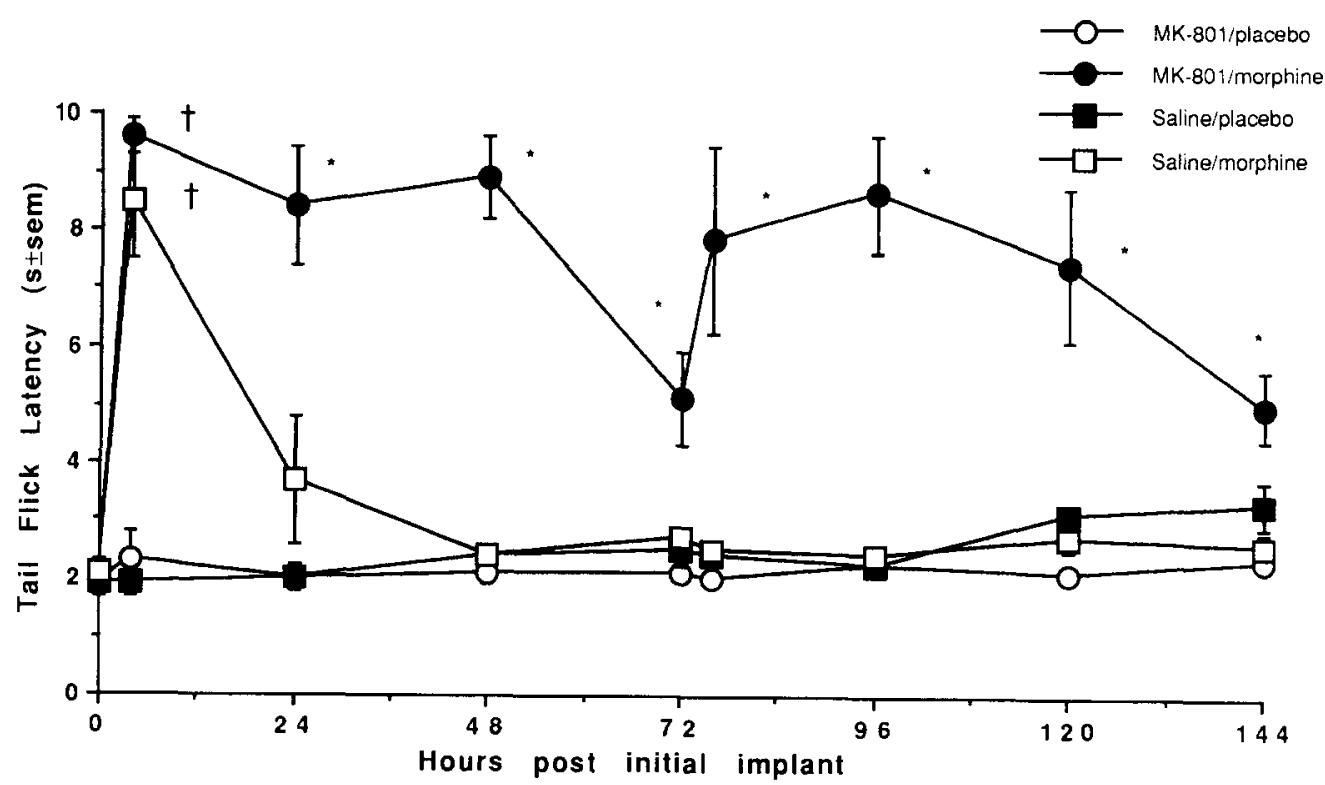

Fig. 1. Effect of MK-801 on morphine tolerance in spinalized rats. Twenty rats underwent $T_{8}$ spinal transection and were allowed to recover for 1 week. Animals then were implanted with osmotic minipumps infusing either MK-801 or saline, and with either 75 mg morphine or placebo pellets. One additional pellet of the same type was implanted 3 days later. Tail flick latencies were tested prior to and $4 \mathrm{~h}$ after each pellet implantation, and daily thereafter. MK-801 exhibited no intrinsic analgesic properties, and did not augment morphine analgesia. Rats treated with saline and morphine rapidly developed morphine tolerance, and did not display analgesia with repeated morphine pelleting. Rats treated with MK-801 and morphine showed significant analgesia throughout the study and an increase in analgesia with repeat pelleting. ${ }^{\dagger} P<0.05$ when compared with placebo groups. ${ }^{*} P<0.05$ compared to placebo and saline-morphine groups. 
The high levels of morphine antinociception seen throughout the experiment in MK-801 treated animals is consistent with earlier suggestions that this drug inhibits the development of opiate tolerance ${ }^{2.11-15}$. The lack of effect of MK-801 in placebo pelleted animals confirms previous work demonstrating that MK-801 itself does not affect the tail-flick response ${ }^{11,14}$. Since MK-801 did not affect tail-flick latencies, the increased antinociception seen in the MK-801/morphine group was not due to MK-801-induced changes in antinociception. Although MK-801 had no effects on its own, it might be argued that this drug could alter the antinociceptive actions of morphine, leading to the observed increases in tail-flick latency. However, although not examined in the present experiment, previous studies have convincingly demonstrated that MK-801 does not alter the antinociceptive effects of morphine ${ }^{13,14,17}$. It thus appears that MK- 801 inhibits the development of tolerance in spinalized animals. The ability of MK-801 to inhibit tolerance in a preparation devoid of supraspinal input provides strong evidence that learned associations did not play a major role in the effects of this drug. The results therefore lead us to conclude that MK- 801 has the ability to inhibit non-associative opiate tolerance at the spinal level. These results support previous findings on the ability of NMDA receptor antagonists to inhibit non-associative opiate tolerance in animals with an intact spinal cord ${ }^{2,17}$.

Previous studies have shown a reduction of baseline tail-flick latency ${ }^{3}$ and a decrease in the maximum antinociceptive effect of morphine ${ }^{1,3}$ in spinalized rats. Although not directly examined in the present experiment, when compared with similar paradigms in intact animals ${ }^{17}$, our results appear to reflect a similar change in the effect of morphine. However, despite the potential differences in the antinociceptive effects of morphine, the inhibition of tolerance seen with MK-801 in spinal animals is at least as robust as the effect seen in intact animals ${ }^{14,17}$. The results suggest that inhibition of neural plasticity in the spinal cord, rather than in the brain, is responsible for the inhibition of tolerance by NMDA receptor antagonists. However, our data do not exclude the possibility that supraspinal sites may contribute to the inhibition of tolerance in the intact animal.

In sum, these data show that MK-801 inhibits the development of morphine tolerance in a preparation devoid of supraspinal input. It thus appears that the effects of NMDA receptor antagonists on opiate tolerance may be primarily due to the ability of these drugs to inhibit non-associative tolerance. NMDA receptors appear to be involved in the development of opiate tolerance at spinal sites, and may play a fundamental physiologic role in this process. These findings may have important implications both for understanding basic mechanisms of tolerance development and for the clinical management of patients requiring long term narcotic therapy.

We would like to thank Dr. Huda Akil for her generous advice, encouragement and financial support, and James Stewart for his excellent technical assistance. This work was supported by NIDA Grant DA02265, the Lucille Markey Charitable Fund, and a Department of Anesthesiology starter grant.

1 Advokat, C. and Gulati, A., Spinal transection reduces both spinal antinociception and CNS concentration of systemically administered morphine in rats, Brain Res., 555 (1991) 251-258.

2 Ben-Eliyahu, S., Marek, P., Vaccarino, A.L., Mogil, J.S., Sternberg, W.F. and Liebeskind, J.C., The NMDA receptor antagonist MK-801 prevents long-lasting non-associative morphine tolerance in the rat, Brain Res., 575 (1992) 304-308.

3 Berge, O.G. and Hole, K., Tolerance to the antinociceptive effect of morphine in the spinal rat, Neuropharmacology, 20 (1981) 653-657.

4 Collingridge, G.L. and Lester, R.A.J., Excitatory amino acid receptors in the vertebrate central nervous system, Pharmacol. Rec., 41 (1989) 143-210.

5 Collingridge, G.L. and Singer, W., Excitatory amino acids and synaptic plasticity, Trends Pharmacol. Sci., 11 (1990) 290-296.

6 D'Amour, F.E. and Smith, D., A method for determining loss of pain sensitization, J. Pharmacol. Exp. Ther., 72 (1941) 74-79.

7 Gutstein, H.B., Bronstein, D.M. and Akil, H., $\beta$-Endorphin processing and cellular origins in rat spinal cord, Pain, 51 (1992) 241-247.

8 Gutstein, H.B., Trujillo, K.A. and Akil, H., Does MK-801 inhibit the development of morphine tolerance in the rat at spinal sites?, Anesthesiology, 77 (1992) A737.

9 Gutstein, H.B., Trujillo, K.A. and Akil, H., MK-801 inhibits the development of morphine tolerance in the rat at spinal sites. Soc. Neurosci. Abstr., 18 (1992) 156.7.

10 Harris, L.S. and Pierson, A.K., Some narcotic antagonists in the benzophan series, J. Pharmacol. Exp. Ther., 143 (1964) 141-148.

11 Marek, P., Ben-Eliyahu, S., Gold, M. and Liebeskind, J.C., Excitatory amino acid antagonists (kynurenic acid and MK-801) attenuate the development of morphine tolerance in the rat. Brain Res., 547 (1991) 77-81.

12 Marek, P., Ben-Eliyahu, S., Vaccarino, A.L. and Liebeskind, J.C., Delayed application of MK-801 attenuates development of morphine tolerance in rats, Brain Res., 558 (1991) 163-165.

13 Trujillo, K.A. and Akil, H., Behavioral interactions between morphine and MK-801: analgesia, tolerance, dependence and lethality, Soc. Neurosci. Abstr., 16 (1990) 211.

14 Trujillo, K.A. and Akil, H., Inhibition of morphine tolerance and dependence by the NMDA receptor antagonist MK-801. Science, 251 (1991) 85-87.

15 Trujillo, K.A. and Akil, H., The NMDA receptor antagonist MK-801 increases morphine catalepsy and lethality, Pharmacol. Biochem. Behav:, 38 (1991) 673-675.

16 Trujillo, K.A. and Akil, H., Opiate tolerance and dependence recent findings and synthesis, New Biol., 3 (1991) 915-923.

17 Trujillo, K.A. and Akil, H., Inhibition of opiate tolerance by non-competitive $N$-methyl-D-aspartate receptor antagonists, submitted. 